\title{
Current Treatments in Diabetic Macular Edema
}

\author{
Archana Seethala ${ }^{1 *}$, Steven Ness ${ }^{2}$ and Manju Subramanian² \\ ${ }^{1}$ Boston Medical Center, 85 East Concord Street, 8th floor, Boston, MA, 02218, USA \\ ${ }^{2}$ Department of Ophthalmology, Boston Medical Center, 85 East Concord Street, 8th floor, Boston, MA, 02218, USA
}

Received: February 12, 2015; Accepted: April 23, 2015; Published: May 07, 2015

*Corresponding author: Archana Seethala, Boston Medical Center, 85 East Concord Street, 8th floor, Boston, MA, 02218, USA, Tel: 5023304349;

Email: archana.seethala@bmc.org

\begin{abstract}
As the incidence of diabetes increases yearly, so does the frequency of vision threatening diabetic retinopathy. The most common cause of vision loss in diabetes is diabetic macular edema. Over the last decade, the gold standard for treatment has transitioned from laser photocoagulation to anti- vascular endothelial growth factor agents. With further understanding of the pathophysiology of this disease and the aide of advanced imaging modalities such as optical coherence tomography and fluorescein angiography, several novel treatment options lie on the horizon. An understanding of options for management of macular edema is essential for providers and patients alike to help achieve best potential outcomes.
\end{abstract}

\section{Introduction}

Diabetes affects an estimated 347 million people worldwide in 2014. According to the World Health Organization, this number is expected to double by 2030. Diabetic Retinopathy (DR), which occurs in approximately $30 \%$ of patients with Diabetes Mellitus (DM), is a leading cause of vision loss in the working age population, 20 to 74 years old [1,2]. This vision loss is most frequently due to Diabetic Macular Edema (DME). In the United States alone, approximately 1 of every 25 persons above the age of 40 with diabetes has DME in at least 1 eye [3].

Both type 1 and type 2 DM patients are susceptible to DME. The likelihood of developing DME increases with severity of retinopathy as well as duration since onset of diabetes [4].

The pathogenesis of DME is primarily due to sustained hyperglycemia causing a breakdown in the blood retinal barrier. Blood vessel walls are composed of tight junctions between retinal vascular endothelial cells. This breakdown of vessel walls causes extravasation of proteins and solutes from the capillaries into the extracellular space, shifting the oncotic and hydrostatic pressure. This leads to fluid in extracellular space, and the eventual formation of DME [5].

Management of DME has evolved a great deal since the 1980's when laser was considered the gold standard of treatment. In the past 10 years intravitreal injections of steroids and antiVascular Endothelial Growth Factor (VEGF) agents have become the mainstays of treatment, changing the way we approach treatment of this disease.

\section{Symptoms}

Patients with DM may present in a variety of ways. If the macular edema does not affect the central vision, or fovea, then many patients are asymptomatic. Others may complain of a gradual decrease in vision with distortion. Transient bilateral decrease in vision is also a symptom of DME, and can be caused by acute changes in blood sugars. A sudden increase in floaters in a patient may be a sign of vitreous hemorrhage in a patient with proliferative diabetic retinopathy. Over time, areas of neovascularization that fibrose and regress, may lead to a tractional retinal detachment. This may be gradual or sudden depending on the portion of the eye affected, and usually leaves patients with a "curtain" in their vision or a portion of their visual field missing. Patients with decreased vision and severe pain may be experiencing neovascular glaucoma due to proliferative diabetic retinopathy. Cataracts can also progress over time in diabetic patients. Patients with cataracts often complain of gradual vision loss with glare around lights and decrease in quality of color vision.

\section{Diagnosis}

Patients with a new diagnosis of diabetes should have a baseline dilated fundus examination by a optometrist or comprehensive ophthalmologist as part of their workup. Patients who are then found to have pathology are referred to a retina specialist for evaluation and possible treatment. In addition to clinical exam, imaging modalities such as fundus photography, optical coherence tomography and fluorescein angiography can aid in the diagnosis of DME.

Intravenous Fluorescein Angiography (IVFA) became popular and accepted in the late 60's. Today, angiography is performed digitally, allowing for real-time evaluation of a patient's retinal circulation. In DR, IVFA can demonstrate both retinal leakage and ischemia, and/or neovascularization especially on wide field angiograms. Angiographic findings in macular edema include progressive leakage from areas of microaneurysmal vascular changes. The most common adverse reactions to occur 
are nausea (3-5\% of patients), vomiting (7\%) of patients), and pruritus. More severe anaphylactic reactions are rare. Patients may experience temporary erythema and mild pain if dye should extravasate during an injection. This is often relieved with an icepack. Local tissue necrosis has been reported in extremely rare cases. However, more commonly patients experience temporary mild pain and redness, which is often relieved with an icepack. Pregnancy is a relative contraindication for this test $[6,7]$.

Optical Coherence Tomography (OCT, is a non-invasive diagnostic tool that obtains in -vivo cross sectional views of the retina. spectral Domain OCT's obtain between 20,000 and 40,000 scans per second, greatly improving resolution, and decreasing likelihood of overlooking pathology [8]. Retinal thickness can be measured and compared from visit to visit to the micron, providing an objective means by which to monitor for progression and treatment response. Specifically, OCT helps to quantify and tract intraretinal and subretinal fluid in patients with DME.

\section{Categories of DME}

During diagnostic evaluation of DME, retina specialists will describe and subsequently categorize DME based on its extent and location. DME can be diffuse or focal (describing extent of the edema), and fovea involving vs. non-fovea involving (describing location of the edema). These categories often aid in determining treatment options.

\section{Treatments}

\section{Medical management}

The Diabetes Complications and Control Trial (DCCT) and United Kingdom Prospective Diabetes Study (UKPDS) demonstrated the importance of glycemic and blood pressure control in preventing both development and progression of DR in Type 1 and 2 diabetes [9]. This remains the best approach for prevention of diabetic retinopathy, and these controls should be prioritized by both the primary care provider as well as the ophthalmologist.

\section{Laser therapy}

Argon laser photocoagulation is the mainstay of therapy for focal, non-fovea involving diabetic macular edema $[9,10]$. It is performed in the clinical setting as an outpatient procedure. Laser was the standard of treatment for DME beginning in the 1980's, and remained the benchmark by which all future treatments were measured until recently. Two landmark studies, the Diabetic Retinopathy Study (DRS) and Early Treatment of Diabetic Retinopathy Study (ETDRS) demonstrated the efficacy of focal laser therapy when applied to non-fovea involving areas of DME. When compared to observation, focal laser resulted in a $50 \%$ reduction in the rate of moderate vision loss at 3 years [11]

One of the major benefits of focal laser compared to the other treatment modalities discussed in this review is that it is a noninvasive treatment with patients experiencing minimal, if any discomfort. These spot scan target any leaking microaneurysms and are applied in a grid like fashion overlying areas of retinal

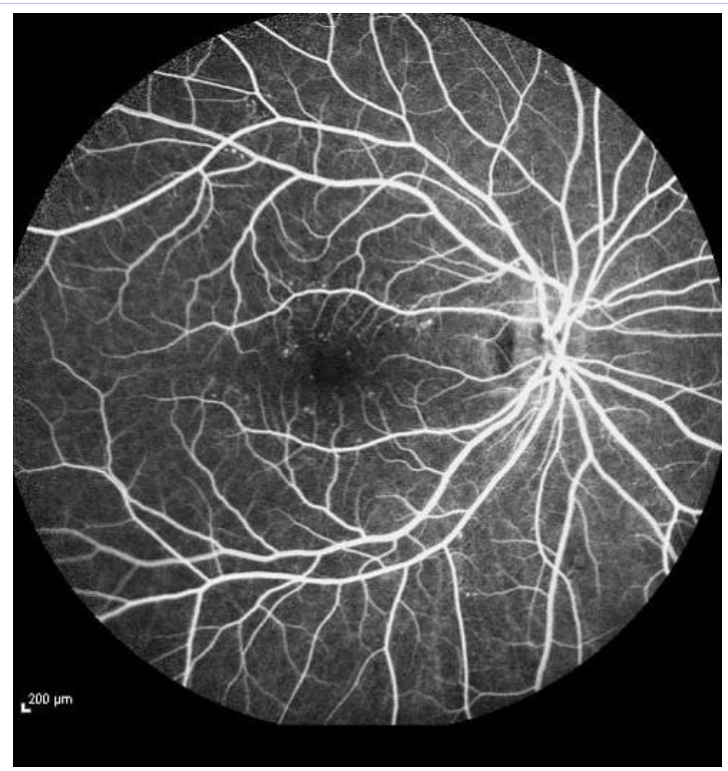

Figure 1a: Early Fluorescein angiogram image of right eye, with punctate areas of hyperfluorescence (arrow) in central macula consistent with microaneurysms found in diabetic retinopathy.

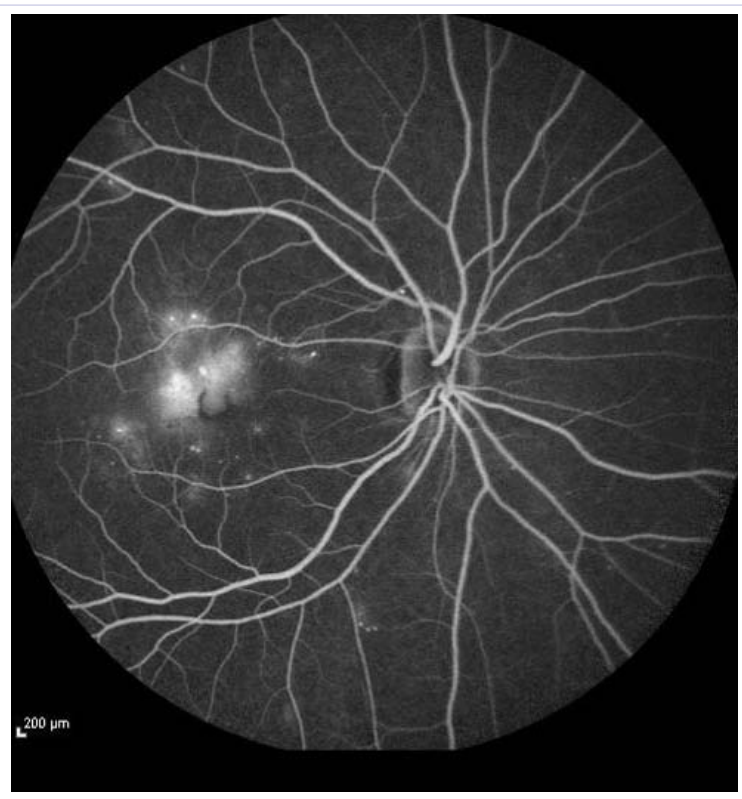

Figure 1b: Late Fluorescein angiogram images ( 9 minutes and 44 seconds after dye injection in same patient) representing larger, brighter areas of hyperfluoresence (arrow) in the central macula representing macular edema and leakage.

thickening. The entire procedure lasts approximately 10-15 minutes, and effects of the laser (decrease in macular edema) are seen approximately 6 weeks afterward. During treatment, care is taken to avoid the foveal center, as laser burns in this location would cause scotomas.

Treatment is recommended for macular edema that meets the criteria for clinical significance,as defined by the ETDRS study [12]. The mechanism by which focal laser decreases DME remains 


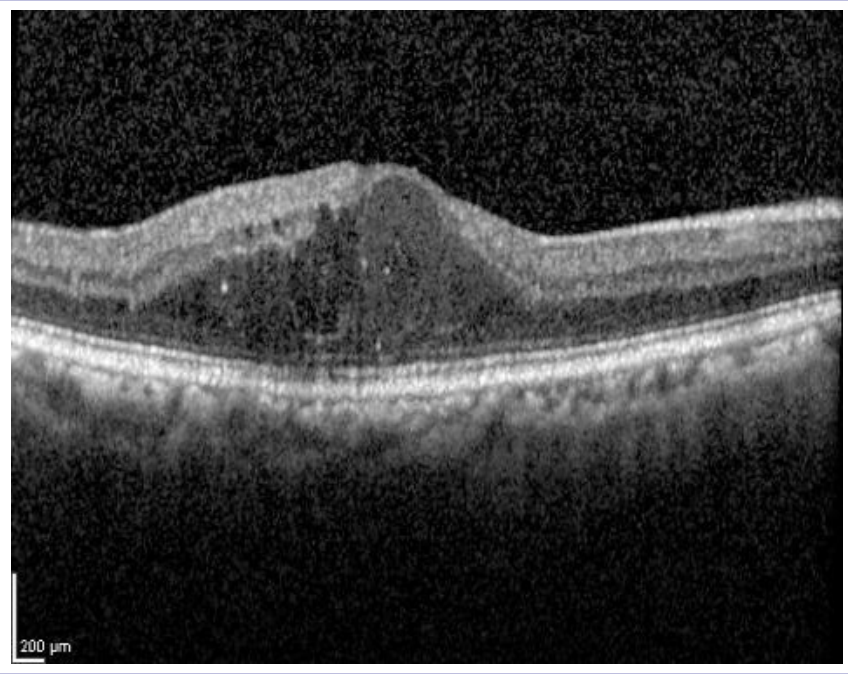

Figure 2a: Optical Coherence tomography of same patient showing disappearance of the foveal pit due to presence of intraretinal fluid with several intraretinal cysts (arrows).

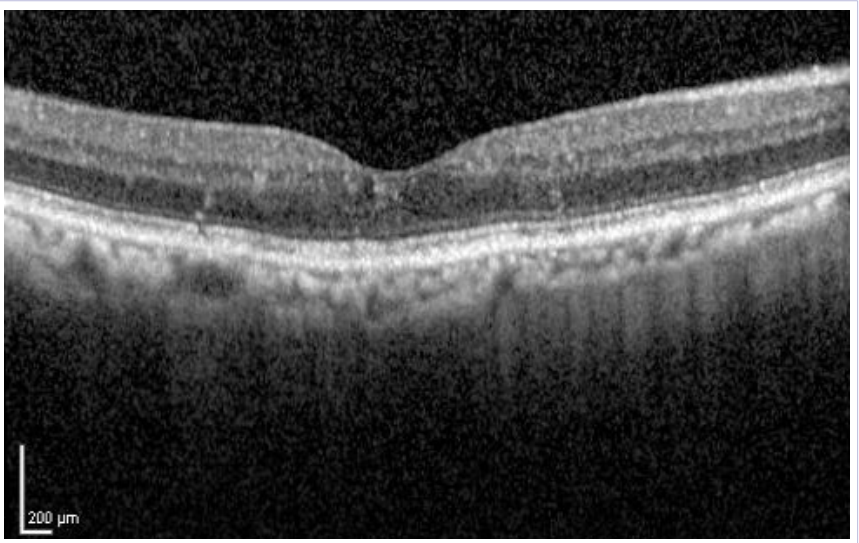

Figure 2b: Post anti-VEGF injection OCT corresponding cross section of retina in same patient, with significant improvement of macular edema and return of foveal depression (arrow head).

unknown, but it is hypothesized that direct photocoagulation of microaneurysms reduces flow and decreases leakage. In addition, laser may stimulate the retinal pigment epithelium, resulting in increased resorption of extracellular fluid [13]. Focal laser can be repeated with caution, should recurrences of macular edema occur, and can be performed in conjunction with any of the other treatments for macular edema. While focal laser remains an excellent treatment option for patients with nonfovea involving DME and can prevent further vision loss, it does not necessarily offer the same potential for visual gain associated with intravitreal therapy [12].

\section{Intravitreal therapy}

Intravitreal therapy is the current gold standard of treatment for cases of focal and diffuse fovea-involving DME. Treatment patterns for DME with intravitreal injections may vary between each retina specialist and even between each patient. Some specialists treat PRN (as needed), and will inject if there is a presence of DME on examination. Others will "load" the patient with a series of 3 monthly injections, followed by a PRN, approach, or a treat and extend approach - during which a patient is treated at each subsequent follow up, but intervals between each follow up gradually increase.

\section{Intravitreal anti-VEGF pathogenesis}

Vascular endothelial growth factor (VEGF) is an essential moderator of blood-retinal barrier breakdown, particularly in regards to angiogenesis and permeability. Hypoxia resulting from microvascular occlusion in DR stimulates the release of VEGF to compensate for lack of perfusion [14]. The finding of elevated vitreous VEGF levels in patients with DME led to the proposal of VEGF inhibitors as a potential treatment for DME.

\section{Administration of medications:}

Intravitreal injections are administered in the clinical setting. While each retina specialist may have slight differences in terms of preparation for an injection, patients can expect to be in a reclined position, with local anesthesia administered to the treatment eye in the form of drops, gel, or subconjunctival injection. A $5 \%$ betadine wash is performed, and a lid speculum is placed to provide adequate exposure. These measures appear to be effective in limiting the incidence of injection - related infection (endophthalmitis), with most studies revealing a 1/2000 risk of this site-threatening complication [15]. The intravitreal injection is performed $4 \mathrm{~mm}$ from the limbus conjunctiva) using a 30 -gauge needle on a $1 \mathrm{cc}$ syringe. Approximately $0.05 \mathrm{cc}$ of medication is injected into the center of the vitreous cavity. Patients do not require a patch after the injection. There is no limit to the number of injections a patient can receive, and most patients require multiple injections at intervals as close as 4 weeks.

Bevacizumab (Avastin, Genentech/Roche): Bevacizumab is a recombinant humanized monoclonal antibody with an immunogenic Fc fragment that blocks VEGF-A, which was first FDA approved for treatment of metastatic colorectal cancer. It is now widely used off-label for the treatment of DME as well as Age Related Macular Degeneration.

Bevacizumab costs approximately $\$ 50$ a dose, much less than its competitor's ranibizumab and aflibercept (priced at $\$ 1800$ 2000 a dose). This price difference, as well as the off-label status of intravitreal bevacizumab for DME, has caused a great deal of debate amongst retina specialists regarding which should be used [15]. Unfortunately, at this point we have no head-to-head trials comparing the 3 medications for DME, although trials, have found bevacizumab and ranibizumab to have equivalent visual acuity outcomes in the treatment of age related macular degeneration[16,17].

Ranibizumab (Lucentis, Genentech): Ranibizumab is a humanized monoclonal antibody fragment that was engineered specifically for ophthalmologic use and was approved by the FDA in 2006 for treatment of age related macular degeneration, and subsequently for DME and retinal vein occlusions., This molecule targets VEGF-A with high affinity. Due to its small size and the fact that it is without an Fc fragment, ranibizumab clears 
from systemic circulation quickly, and is thought to have the least systemic absorption when compared to the other antivegf alternatives. Several studies including those by the DRCR net [18], the RESOLVE study [19], and the RISE and RIDE [20] trials confirmed ranibizumab's effectiveness in the treatment of DME, with resulting visual acuity gains.

Aflibercept (Eylea, Regeneron Pharmaceuticals): The most recently developed of the anti VEGF medications, aflibercept is composed of key domains from human VEGF receptors 1 and 2 fused to the Fc domain of human immunoglobulin G1. It's binding affinity to VEGF-A is $100 x$ greater than bevacizumab or ranibizumab [16]. Animal studies have shown aflibercept to have a longer intraocular half-life than bevacizumab and ranibizumab, potentially allowing for decreased injection frequency [17]. Initially used for exudative age-related macular degeneration, aflibercept is now FDA approved for use in central and branch retinal vein occlusions, and as late as 2014, for use in DME.

Which is better? Recently, the 1 year results of Protocole $\mathrm{T}$, a study comparing the efficacy of previously discussed antivegf agents (Bevacizumab, Ranibizumab, and Aflibercept) were published in the New England Journal of Medicine [21]. While it was concluded that all three agents provided patients with substantial visual acuity improvement within 1 month of use and over the first year, on average aflibercept offered patients greater improvement. This improvement however, was relative to presenting visual acuity. Patients with visual acuity better than $20 / 40$ on presentation showed very little difference in response to any of the aforementioned agents. Patients with worse initial vision loss (worse than 20/50) had an advantage when using aflibercept. As further information arises from more long term follow up data, adjustments may be made to current treatment algorithms.

Risks discussed with any intravitreal injection include mainly the risk of infection, but also risk of vitreous hemorrhage, inflammation, retinal tears or detachment. All agents also are absorbed systemically, however ranibizumab, due to its relatively short systemic half life, is thought to absorb less. This may influence some ophthalmologists to tailor which agent is used for each individual patient, given history of recent cardiovascular health issues (heart attack, stroke, clotting disorders, etc).

The CATT trial [22] specifically found that the rate of infection between avastin and lucentis when administered as intravitreal injections was similar (approx.1/2000). It also demonstrated that there were similar rates of death, heart attack, and stroke with the two medications. It did find that the rates of hospitalization due to serious events were higher in the group receiving avastin, but that was unclear if it would have been similar in untreated patients with similar comorbidities as well.

Corticosteroids: Inflammation has also been shown to play a significant role in the development of diabetic macular edema [23]. As a result,corticosteroids with both anti-inflammatory and anti-VEGF properties have also been utilized in the treatment of DME[24]. Triamcinolone acetonide can be injected intravitreally in the form of Kenalog (Bristol-Myers Squibb), Triescence
(Alcon), or Trivaris (Allergan). Although Triescence and Trivaris are FDA approved for intravitreal use in treatment of intraocular inflammation, their use for diabetic macular edema remains off label.

Ocular side-effects of intravitreal corticosteroid use include cataract formation and intraocular pressure elevation, which occur at significantly greater frequency than with intravitreal anti-VEGF agents [25,26]. Because of these concerns, steroids have decreased in popularity, and now are used as a second or third line therapy or in combination with laser or anti-VEGF agent for refractory cases of DME.

Sustained steroid-releasing intravitreal implants placed in the clinical setting, are also an option in treating DME. Ozurdex (Allergan) is a dexamethasone implant that is biodegradable and lasts for 3-6 months. Insertion technique is similar to that previously described with intravitreal injections. Studies of Illuvien (Fluocinolone, Alimera Sciences) have demonstrated sustained visual acuity gains for 36 months with a single intravitreal insert [27]. The benefit of decreased dosing intervals with corticosteroid implants must be balanced with their associated cataractogenic properties, although they may be an excellent option in patients who have already undergone cataract surgery [28]. Intraocular pressure must be carefully monitored in these patients as one of the most concerning side effects of peri and intraocular steroid therapy is acute elevations in pressure.

Surgical treatment: Traction from interface abnormalities between the vitreous and retina have been considered a potential source of DME, and thus in refractory cases of DME where all other options have been exhausted, pars plana vitrectomy is considered. In addition to relieving vitreomacular traction forces, vitrectomy surgery also increases intraocular oxygen levels and thus may decrease ischemia driven VEGF secretion [29]. Studies of vitrectomy surgery with epiretinal and/or inner limiting membrane peeling have shown decreases in central retinal thickness and in some cases improved visual acuity outcomes $[30,31]$. Though a good option in certain cases, the risk versus benefit of surgery must be considered.

\section{The Future}

There are many exciting ongoing investigations in the field of diabetic macular edema. New treatments aim to increase treatment intervals, decrease risks of intravitreal and surgical procedures, and most importantly improve outcomes for patients. Topical medications in the form of drops, such as Dexamethasone-Cyclodextrin, which is a steroid with excellent intraocular penetration, as well as Mecamylamine, which is an antagonist of nACh are currently being investigated [32,33]. Subconjunctival injections are also being considered using mTOR inhibitors such as Sirolimus and rapamycin [34].

Danazol (Optina) is an oral androgen, which has shown to enhance endothelial barrier function and decrease vascular permeability in preclinical in vitro studies on human endothelial cells [35] and is currently being tested in human trials. Intravenous medications are also under investigation for refractory cases of diabetic macular edema. 


\section{Conclusions}

As the number of patients diagnosed with diabetes continues to increase, so do the number of patients with macular edema and visual impairment. Monumental advances have been made in the recent past that have given patients and ophthalmologists alike an arsenal of options with which to preserve and improve vision. Even more promising therapeutic options are on the horizon.

\section{References}

1. Moss SE, Klein R, Klein BE. The 14-year incidence of visual loss in a diabetic population. Ophthalmology. 1998; 105(6):998-1003.

2. Yau JW, Rogers SL, Kawasaki R, Lamoureux EL, Kowalski JW, Bek T, et al. Global prevalence and major risk factors of diabetic retinopathy. Diabetes Care. 2012; 35(3):556-564. doi: 10.2337/dc11-1909.

3. Varma R, Bressler NM, Doan QV, Gleeson M, Danese M, Bower JK, et al. Prevalence of and risk factors for diabetic macular edema in the United States. JAMA Ophthalmol. 2014; 132(11):1334-1340. doi: 10.1001/jamaophthalmol.2014.2854.

4. Bhagat N, Grigorian RA, Tutela A, Zarbin MA. Diabetic macular edema: pathogenesis and treatment. Surv Ophthalmol. 2009; 54(1):1-32. doi: 10.1016/j.survophthal.2008.10.001.

5. Zhang X, Zeng H, Bao S, Wang N, Gillies MC. Diabetic macular edema: new concepts in patho-physiology and treatment. Cell \& bioscience. 2014; 4:27. doi: 10.1186/2045-3701-4-27.

6. Gass JD, Sever RJ, Sparks D, Goren J. A combined technique of fluorescein funduscopy and angiography of the eye. Arch Ophthalmol. 1967; 78(4):455-461.

7. Yannuzzi LA, Rohrer KT, Tindel LJ, Sobel RS, Costanza MA, Shields W, et al. Fluorescein angiography complication survey. Ophthalmology. 1986; 93(5):611-617.

8. Huang D, Swanson EA, Lin CP, Schuman JS, Stinson WG, Chang W, et al. Optical coherence tomography. Science. 1991; 254(5035):1178-1181.

9. Robert Turner, Rury Holman, Irene Stratton, Carole Cull, Valeria Frighi, Susan Manley, et al. Tight blood pressure control and risk of macrovascular and microvascular complications in type 2 diabetes: UKPDS 38. UK Prospective Diabetes Study Group. Bmj. 1998; 317(7160):703-713.

10. Blankenship GW. Diabetic macular edema and argon laser photocoagulation: a prospective randomized study. Ophthalmology. 1979; 86(1):69-78.

11.Writing Committee for the Diabetic Retinopathy Clinical Research Network. Fong DS, Strauber SF, Aiello LP, Beck RW, Callanan DG, Danis RP, et al. Comparison of the modified Early Treatment Diabetic Retinopathy Study and mild macular grid laser photocoagulation strategies for diabetic macular edema. Arch Ophthalmol. 2007; 125(4):469-480.

12. Photocoagulation for Diabetic Macular Edema. Early Treatment Diabetic Retinopathy Study report number 1. Early Treatment Diabetic Retinopathy Study research group. Arch Ophthalmol. 1985; 103(12):1796-1806. doi:10.1001/archopht.1985.01050120030015.

13. Lee CM, Olk RJ. Modified grid laser photocoagulation for diffuse diabetic macular edema. Long-term visual results. Ophthalmology. 1991; 98(10):1594-1602.

14. Funatsu H, Yamashita H, Noma H, Mimura T, Yamashita T, Hor S. Increased levels of vascular endothelial growth factor and interleukin- 6 in the aqueous humor of diabetics with macular edema. Am J Ophthalmol. 2002; 133(1):70-77.

15. Dakin HA, Wordsworth S, Rogers CA, Abangma G, Raftery J, Harding $\mathrm{SP}$, et al. Cost-effectiveness of ranibizumab and bevacizumab for age-related macular degeneration: 2-year findings from the IVAN randomised trial. BMJ open. 2014; 4(7):e005094. doi:10.1136/ bmjopen-2014-005094.

16. Papadopoulos N, Martin J, Ruan Q, Rafique A, Rosconi MP, Shi E, et al. Binding and neutralization of vascular endothelial growth factor (VEGF) and related ligands by VEGF Trap, ranibizumab and bevacizumab. Angiogenesis. 2012; 15(2):171-85. doi: 10.1007/ s10456-011-9249-6.

17. Do DV, Schmidt-Erfurth U, Gonzalez VH, Gordon CM, Tolentino M Berliner AJ, et al. The DA VINCI Study: phase 2 primary results of VEGF Trap-Eye in patients with diabetic macular edema. Ophthalmology. 2011; 118(9):1819-1826. doi: 10.1016/j.ophtha.2011.02.018.

18. Diabetic Retinopathy Clinical Research N, Scott IU, Edwards AR, Beck RW, Bressler NM, Chan CK, Elman MJ, et al. A phase II randomized clinical trial of intravitreal bevacizumab for diabetic macular edema. Ophthalmology. 2007; 114(10):1860-1867.

19. Massin P, Bandello F, Garweg JG, Hansen LL, Harding SP, Larsen M, et al. Safety and efficacy of ranibizumab in diabetic macular edema (RESOLVE Study): a 12-month, randomized, controlled, doublemasked, multicenter phase II study. Diabetes care. 2010; 33(11):23992405. doi: 10.2337/dc10-0493.

20. Nguyen QD, Brown DM, Marcus DM, Boyer DS, Patel S, Feiner L, et al. Ranibizumab for diabetic macular edema: results from 2 phase III randomized trials: RISE and RIDE. Ophthalmology. 2012 Apr;119(4):789-801. doi: 10.1016/j.ophtha.2011.12.039.

21. Diabetic Retinopathy Clinical Research N, Wells JA, Glassman AR, Ayala AR, Jampol LM, Aiello LP, et al. Aflibercept, Bevacizumab, Or Ranibizumab for Diabetic Macular Edema. N Engl J of Med. 2015; 372(13):1193-1203. DOI: 10.1056/NEJMoa1414264.

22. Comparison of Age-related Macular Degeneration Treatments Trials Research G, Martin DF, Maguire MG, Fine SL, Ying GS, Jaffe GJ, Grunwald JE, et al. Ranibizumab and bevacizumab for treatment of neovascular age-related macular degeneration: two-year results. Ophthalmology. 2012; 119(7):1388-1398. doi: 10.1016/j.ophtha.2012.03.053.

23. Funatsu H, Noma H, Mimura T, Eguchi S, Hori S. Association of vitreous inflammatory factors with diabetic macular edema. Ophthalmology. 2009; 116(1):73-9. doi: 10.1016/j.ophtha.2008.09.037.

24. Nauck M, Karakiulakis G, Perruchoud AP, Papakonstantinou E, Roth M. Corticosteroids inhibit the expression of the vascular endothelial growth factor gene in human vascular smooth muscle cells. Eur J Pharmacol. 1998; 341(2-3):309-315.

25. Bandello F, Preziosa C, Querques G, Lattanzio R. Update of intravitreal steroids for the treatment of diabetic macular edema. Ophthalmic Res. 2014; 52(2):89-96. doi: 10.1159/000362764.

26.Zacharias LC, Lin T, Migon R, Ghosn C, Orilla W, Feldmann B, et al. Assessment of the differences in pharmacokinetics and pharmacodynamics between four distinct formulations of triamcinolone acetonide. Retina. 2013 Mar;33(3):522-31. doi: 10.1097/IAE.0b013e3182647f69.

27. Campochiaro PA, Brown DM, Pearson A, Chen S, Boyer D, Ruiz-Moreno J, et al. Sustained delivery fluocinolone acetonide vitreous inserts provide benefit for at least 3 years in patients with diabetic macular edema. Ophthalmology. 2012 Oct;119(10):2125-32. doi: 10.1016/j. ophtha.2012.04.030. 
28. Haller JA, Kuppermann BD, Blumenkranz MS, Williams GA, Weinberg DV, Chou C, et al. Randomized controlled trial of an intravitreous dexamethasone drug delivery system in patients with diabetic macular edema. Arch Ophthalmol. 2010 Mar;128(3):289-96. doi: 10.1001/archophthalmol.2010.21.

29. Holekamp NM, Shui YB, Beebe DC. Vitrectomy surgery increases oxygen exposure to the lens: a possible mechanism for nuclear cataract formation. Am J Ophthalmol. 2005 Feb;139(2):302-310.

30. Diabetic Retinopathy Clinical Research Network Writing C, Haller JA, Qin H, Apte RS, Beck RR, Bressler NM, et al. Vitrectomy outcomes in eyes with diabetic macular edema and vitreomacular traction. Ophthalmology. 2010; 117(6):1087-1093.e3. doi: 10.1016/j. ophtha.2009.10.040.

31. Bonnin S, Sandali O, Bonnel S, Monin C, El Sanharawi M. Vitrectomy with internal limiting membrane peeling for tractional and nontractional diabetic macular edema Long-term Results of a Comparative Study. Retina. 2015; 35(5):921-928. doi: 10.1097/IAE.0000000000000433.
32. Campochiaro PA, Shah SM, Hafiz G, Heier JS, Lit ES, Zimmer-Galler I, et al. Topical mecamylamine for diabetic macular edema. Am J Ophthalmol. 2010; 149(5):839-851.e1. doi: 10.1016/j.ajo.2009.12.005.

33. Tanito M, Hara K, Takai Y, Matsuoka Y, Nishimura N, Jansook P, et al. Topical dexamethasone-cyclodextrin microparticle eye drops for diabetic macular edema. Invest Ophthalmol Vis Sci. 2011; 52(11):7944-7948. doi: 10.1167/iovs.

34. Krishnadev N, Forooghian F, Cukras C, Wong W, Saligan L, Chew EY, et al. Subconjunctival sirolimus in the treatment of diabetic macular edema. Graefe's archive for clinical and experimental ophthalmology = Albrecht von Graefes Archiv fur klinische und experimentelle Ophthalmologie. 2011; 249(11):1627-1633.

35. Thomas GW, Rael LT, Bar-Or R, Mains CW, Slone DS, Boyd SR, et al. Biphasic effect of danazol on human vascular endothelial cell permeability and f-actin cytoskeleton dynamics. Biochem Biophys Res Commun. 2012; 421(4):707-712. doi: 10.1016/j.bbrc.2012.04.066. 